Trauma Berufskrankh 2017 19 (Suppl 2):S214-S219 DOI 10.1007/s10039-017-0282-6

Online publiziert: 26. Juli 2017

(c) Springer Medizin Verlag GmbH 2017

CrossMark

\author{
I. M. Mehling ${ }^{2} \cdot$ A. Arsalan-Werner ${ }^{1} \cdot$ M. Sauerbier ${ }^{1}$ \\ ${ }^{1}$ Abteilung für Plastische, Hand- und Rekonstruktive Chirurgie, Berufsgenossenschaftliche Unfallklinik \\ Frankfurt am Main, Frankfurt am Main, Deutschland \\ ${ }^{2}$ Sektion Handchirurgie, St. Vinzenz-Krankenhaus Hanau gGmbH, Hanau, Deutschland
}

\title{
Behandlung von karpalen und metakarpalen Pseudarthrosen
}

\section{Karpale Pseudarthrosen}

gen nach Finger- und Mittelhandfrakturen führen zu einer Beeinträchtigung der Greiffunktion. Bei fehlender knöcherner Heilung kommt es zur Entwicklung einer Pseudarthrose. Pseudarthrosen an der Hand führen zu einer Funktionseinschränkung und persistierenden Schmerzen. An der Handwurzel kann es im weiteren Verlauf zu einer Instabilität mit Gefügestörung bis hin zum karpalen Kollaps kommen [1, 3].

Eine Pseudarthrose gilt als eine nicht geheilte Fraktur, die ohne operative Intervention kein Potenzial zur Heilung zeigt. In der Regel wird eine Pseudarthrose nach fehlender knöcherner Heilung nach 6 Monaten nach Frakturereignis diagnostiziert. In der Literatur wird dies jedoch bei nicht geheilten Skaphoidfrakturen bereits nach 3 Monaten beschrieben [2, 7].

Die Therapie von Pseudarthrosen am Karpus sowie Metakarpus besteht zum einen in der Ausräumung der Pseudarthrose sowie der Auffüllung mittels Spongiosa. Die Spongiosa kann z. B. aus dem Beckenkamm oder dem distalen Radius entnommen werden. In manchen Fällen ist jedoch auch die Anwendung vaskularisierter Knochenspäne indiziert. Nach der Knochenauffüllung ist eine stabile Osteosynthese notwendig, im besten Falle eine belastungsstabile Osteosynthese mit nachfolgender frühfunktioneller Behandlung.

Dieser Übersichtsbeitrag beschreibt die Behandlung karpaler und metakarpaler Pseudarthrosen.
Die häufigste Form der Pseudarthrose am Karpus ist die Skaphoidpseudarthrose. Seltener kommen auch andere Formen wie Pseudarthrosen am Os hamatum, Os capitatum sowie Os lunatum vor.

\section{Skaphoidpseudarthrose}

Die häufigsten Ursachen für eine Skaphoidpseudarthrose sind eine primär übersehende Fraktur, eine unzureichende Immobilisation, eine starke Fragmentdislokation ohne oder mit karpaler Gefügestörung sowie die proximale Skaphoidfraktur. Da der proximale Skaphoidpol keine eigenständige Blutversorgung aufweist, kommt es bei proximalen Skaphoidfrakturen gehäuft zu Pseudarthrosen und avaskulären Nekrosen. Die Skaphoidpseudarthroserate wird in der Literatur zwischen 5 und $15 \%$ beschrieben $[1,4,5,8]$. Im weiteren Verlauf kommt es nach Pseudarthrosenbildung zu einer periskaphoidalen Arthrose mit Ausbildung eines sog. SNAC („scaphoid-nonunion advanced collaps")-Wrist [8].

Klinisch haben die Patienten mit einer Skaphoidpseudarthrose Schmerzen bei Radialabduktion und geben häufig einen Stauchungsschmerz des Daumenstrahls an. Im weiteren Verlauf kommt es bei zusätzlich bestehender karpaler Arthrose zu belastungsabhängigen Schmerzen im Handgelenkbereich sowie zu einer Bewegungseinschränkung mit zunehmender Schwellung im Handgelenk.

Zielführend für eine Skaphoidpseudarthrose ist nach anamnestisch-klinischem Verdacht eine adäquate bildgebende Diagnostik. Zunächst sollte die
Basisdiagnostik des Kahnbeins mittels nativ-radiologischer Untersuchung in dorsopalmarer und seitlicher Projektion in Neutralstellung erfolgen sowie die Aufnahme nach Stecher durchgeführt werden. Zur weiteren Diagnostik sollte ebenfalls zeitnah eine hochauflösende Dünnschicht-Computertomographie (CT)-Untersuchung in schräg-sagittalen Schichten parallel zur Längsachse des Skaphoids erfolgen.

Zur besseren Beurteilung der medullären und ossären Vitalität v. a. des proximalen Kahnbeinfragmentes sollte ggf. ergänzend eine Magnetresonanztomographie (MRT)-Untersuchung mit Kontrastmittelgabe durchgeführt werden.

\section{Einteilung der Skaphoid- pseudarthrose}

Nach Herbert werden Skaphoidfrakturen mit verzögerter knöcherner Heilung („,delayed union“) als Typ C bezeichnet. Beim Typ C zeigt sich radiologisch ein Resorptionsband an beiden Fragmenten parallel zum Frakturspalt. Dieses Stadium ist potenziell reversibel.

Zeigt sich keine erkennbare knöcherne Heilung, so besteht eine Pseudarthrose des Typ D („established nonunion“) [9].

Typ D wird in 4 Subtypen unterschieden:

- D1: straffe Pseudarthrose, gekennzeichnet durch eine stabile bindegewebige Überbrückung, ohne karpale Deformität,

- D2: Pseudarthrosespalt ohne Bindegewebe mit instabilen Fragmenten und beginnender Deformität,

- D3: Sklerosierung entlang des Pseudarthrosespaltes und zunehmende Deformität, 

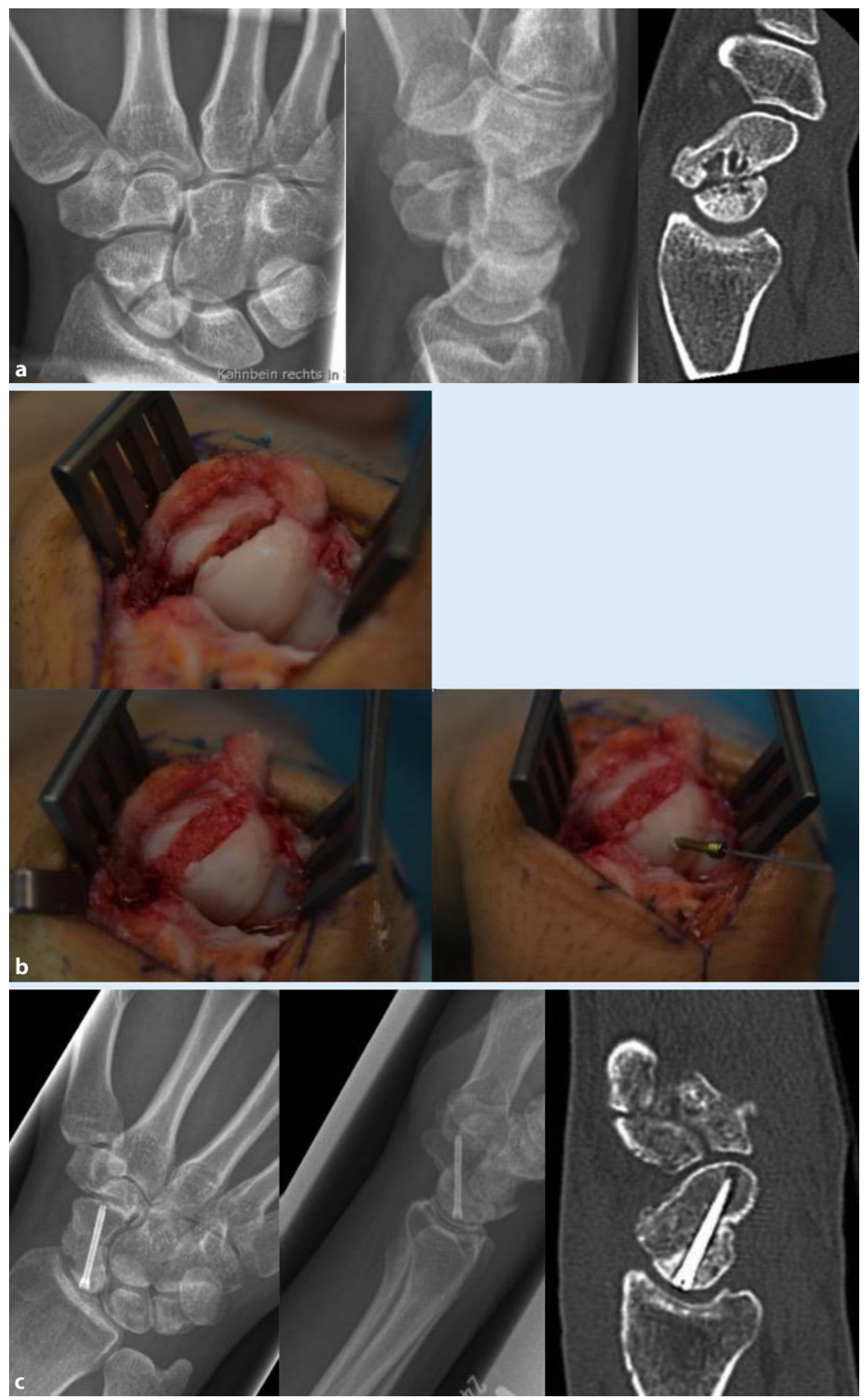

Abb. 1 A Fallbeispiel 1. a Röntgenbilder rechtes Skaphoid in p.-a. und seitlicher Aufnahme sowie Dünnschicht-Computertomographie (CT)-Aufnahme in Längsrichtung zum Skaphoid eines 28-jährigen Patienten mit einer Skaphoidpseudarthrose im mittleren Drittel (Typ D3 nach Herbert) rechtes Handgelenk mit radioskaphoidaler Knorpelschädigung (SNAC [„scaphoid-non-union advanced collaps"]-Wrist Stadium I). b Intraoperative Aufnahmen nach Ausräumung der Pseudarthrose im proximalen Drittel sowie Auffüllung mit Radiusspongiosa und Schraubenosteosynthese. c Postoperative Röntgenverlaufsaufnahmen: Es erfolgte zusätzlich die Resektion des Processus styloideus radii, die Skaphoidrekonstruktion mit Spongiosaplastik vom ipsilateralen distalen Radius und Schraubenosteosynthese mittels kanülierter, selbstbohrender und selbstschneidender Schraube (CCS-Schraube SpeedTip 2,2 mm, APTUS, Medartis AG, Basel, Schweiz). Die Verlaufs-CT-Untersuchung nach 3 Monaten zeigt die regelrechte Schraubenlage bei knöcherner Heilung
- D4: Pseudarthrose mit avaskulärem proximalem Fragment und karpaler Deformität.

\section{Therapie}

Die Therapie der Skaphoidpseudarthrose ist in der Regel eine operative Therapie. Dabei sind die therapeutischen Möglichkeiten zum einen abhängig vom Stadium der Pseudarthrose, zum anderen aber auch im Wesentlichen davon, ob das proximale Fragment perfundiert ist.

Bei einer guten Perfusion sowie einer Pseudarthrose im mittleren Drittel kann eine Spongiosaplastik nach MattiRusse durchgeführt werden. Über einen palmaren Zugang wird ein kortikospongiöser Span zwischen den ausgehöhlten Pseudarthrosespalt eingebracht. Der kortikospongiöse Span wird meist aus dem Beckenkamm oder aus dem distalen Radius entnommen. Bei ausgeprägter Fragmentdislokation werden zusätzlich zur Fixierung Kirschner-Drähte verwendet.

Da sich in den letzten Jahren die Skaphoidosteosynthese kontinuierlich weiterentwickelt hat und nach dem bewährten Prinzip der Doppelgewindeschraube nach Herbert nun selbstbohrende und selbstschneidende Schrauben zur Verfügung stehen, kommt den formwiederherstellenden Verfahren aktuell weiterhin die größte Bedeutung zu.

Hierbei erfolgen zunächst die Ausräumung der Pseudarthrose sowie die Anfrischung des Knochens. Je nach Defektgröße wird dann mittels Spongiosa (entweder aus dem distalen Radius oder dem Beckenkamm) oder mittels Einbringen eines kortikospongiösen Blockes (meist aus dem Beckenkamm) der Defekt aufgefüllt (Fallbeispiel 1, • Abb. 1a-c).

Bei Pseudarthrosen im mittleren Skaphoiddrittel bevorzugen wir den palmaren Zugang, bei Pseudarthrosen im Bereich des proximalen Drittels den dorsalen Zugang. Die Fixierung erfolgt meist mittels kanülierter, selbstbohrender und selbstschneidender Doppelgewindeschraube. Die Osteosynthese mittels Skaphoidplatte kommt dann zum Einsatz, wenn eine Skaphoidrekonstruktion mit Schraubenosteosynthese aufgrund der schlechten knöchernen Struktur des Skaphoids technisch nicht möglich ist (Fallbeispiel 2, • Abb. 2a-b). 
Die Ruhigstellungsdauer beträgt ca. 6 bis 8 Wochen.

Bei minderperfundierten Skaphoidanteilen oder nach fehlgeschlagenen Voreingriffen mit persistierender Pseudarthrose sind revaskularisierende Maßnahmen indiziert. Hierbei kommen gefäßgestielte kortikospongiöse Knochenblöcke z. B. aus dem Radius zur Anwendung. Eine weitere Möglichkeit besteht in der Verwendung freier vaskularisierter Knochentransplantate wie der freien Femurkondylentransplantation [10-14, 20,21].

Besteht die Skaphoidpseudarthrose jedoch schon $\mathrm{zu}$ lange und ist es bereits zu einer karpalen Gefügestörung der Handwurzel im Sinne eines SNACWrist mit karpalem Kollaps gekommen, können nur noch sog. Rettungsoperationen durchgeführt werden [16]. Je nach Stadium erfolgt hierbei eine Resektion des Proc. styloideus radii oder im weiteren Verlauf eine Teilversteifung im Sinne einer mediokarpalen Teilarthrodese sowie einer "proximal row carpectomie“ (PRC) oder - bei vollständig aufgebrauchten Gelenkflächen zwischen dem distalen Radius und dem Karpus - eine komplette Handgelenkversteifung [16].

\section{Pseudarthrose des Os hamatum}

Es handelt sich hierbei um die zweithäufigste Pseudarthroseform am Karpus. Diese ist deutlich seltener als am Skaphoid. Meist ist der Hamulus ossis hamati betroffen [6]. Ursache hierfür ist oft eine unzureichende Immobilisation nach stattgehabter Fraktur oder übersehener Fraktur [6].

Die Therapie besteht bei entsprechender klinischer Symptomatik in der Exzision des Hamulus ossis hamati (Fallbeispiel 3, Abb. 3a-c).

\section{Pseudarthrosen des Os capitatum und Os lunatum}

Diese Pseudarthroseformen sind sehr selten. Meist sind sie Folgen von stattgehabten Hyperextensionsverletzungen sowie nach karpalen Luxationsfrakturen. Oft wird die primäre karpale Fraktur dabei übersehen.

Bei anamnestischem sowie klinischem Verdacht sollte daher stets eine genaue radiologische Diagnostik erfolgen. Diese beinhaltet zunächst eine konventionelle Röntgenbildgebung des Handgelenks in 2 Ebenen. Nachfolgend sind eine Dünnschicht-CT-Untersuchung der Handwurzel sowie ggf. eine MRT-Untersuchung zur weiteren Abklärung indiziert [6].

Die Therapie ist zum einen abhängig von den Beschwerden des Patienten und zum anderen von der Fragmentgröße. Bei kleinen pseudarthrotischen Fragmenten mit bestehender Schmerzsymptomatik kann eine Exzision des Fragmentes erfolgen. Bei größerem Fragment und entsprechender Beschwerdesymptomatik ist nach Ausräumung der Pseudarthrose ggf. eine Auffüllung mittels Spongiosa und Schraubenosteosynthese notwendig.

\section{Metakarpale Pseudarthrosen}

Die Inzidenz einer Pseudarthrose nach Mittelhandknochenfrakturen ist unklar und wird in der Literatur zwischen 0 und $15 \%$ beschrieben. Es handelt sich wie auch bei den karpalen Pseudarthrosen um einen gestörten Heilungsprozess nach stattgehabter Fraktur. Risikofaktoren hierfür sind eine nicht korrekte Frakturreposition, eine instabile bzw. insuffizient durchgeführte Osteosynthese und eine ausgeprägte Weichteilschädigung [7, 17].

Die Pseudarthroserate nach stattgehabter metakarpaler Fraktur variiert je nach Frakturverlauf. So wird nach Querfrakturen im Diaphysenbereich eine Pseudarthroserate von bis zu $30 \%$ beschrieben, wohingegen die Pseudarthroserate bei schrägen Mittelhandknochenbrüchen mit ca. $7 \%$ beschrieben wird [7]. Manuell tätige Arbeiter entwickeln zudem eher eine Pseudarthrose. Ebenso erhöht ein Nikotinabusus das Pseudarthroserisiko erheblich [7].

Wie auch bei anderen knöchernen Lokalisationen von Pseudarthrosen kann die Pseudarthrose an den Mittelhandknochen in eine hypertrophe und eine atrophe Form unterteilt werden. Die hypertrophe Pseudarthrose ist meist durch eine instabile Osteosynthese bedingt. Hieraus ergibt sich auch die entsprechende Therapieform - nämlich die operative
Trauma Berufskrankh 2017 · 19 (Suppl 2):S214-S219

DOI 10.1007/s10039-017-0282-6

๑) Springer Medizin Verlag GmbH 2017

I. M. Mehling · A. Arsalan-Werner .

M. Sauerbier

\section{Behandlung von karpalen und metakarpalen Pseudarthrosen}

\section{Zusammenfassung}

Pseudarthrosen am Karpus und Metakarpus der Hand können zu einer deutlichen Funktionseinschränkung mit persistierenden Schmerzen und fortschreitender Arthrose führen. Die Pseudarthrose des Skaphoids ist die häufigste Pseudarthroseform am Karpus. Metakarpale Pseudarthrosen haben, abhängig von den Risikofaktoren, eine Inzidenz von 0-15\%. Zur Diagnostik und Therapieplanung ist insbesondere bei der Skaphoidpseudarthrose eine differenzierte Bildgebung mit Röntgen und Computertomographie zu fordern. Als Therapie erfolgen meist eine Ausräumung der Pseudarthrose, die Auffüllung mittels nicht vaskularisierter Spongiosa oder vaskularisierten Knochenspänen sowie eine stabile Osteosynthese.

\section{Schlüsselwörter}

Skaphoidpseudarthrose - Osteosynthese .

Schmerzen · Bildgebung · Funktionseinschränkung

\section{Treatment of carpal and metacarpal non-union}

\section{Abstract}

The development of non-union following carpal or metacarpal fractures can lead to severe impairment of hand function with persistent pain and arthritis. Non-union of the scaphoid is the most common form affecting the carpus. Metacarpal non-union has an incidence of $0-15 \%$ depending on the underlying risk factors. For diagnosis, classification and treatment planning computed tomography (CT) scans are mandatory in scaphoid non-unions. The surgical strategy mostly consists of resection of the bony non-union, augmentation using non-vascularized cancellous bone or vascularized autologous bone graft and a stable osteosynthesis.

\section{Keywords}

Scaphoid non-union - Osteosynthesis - Pain . Imaging · Functional impairment 

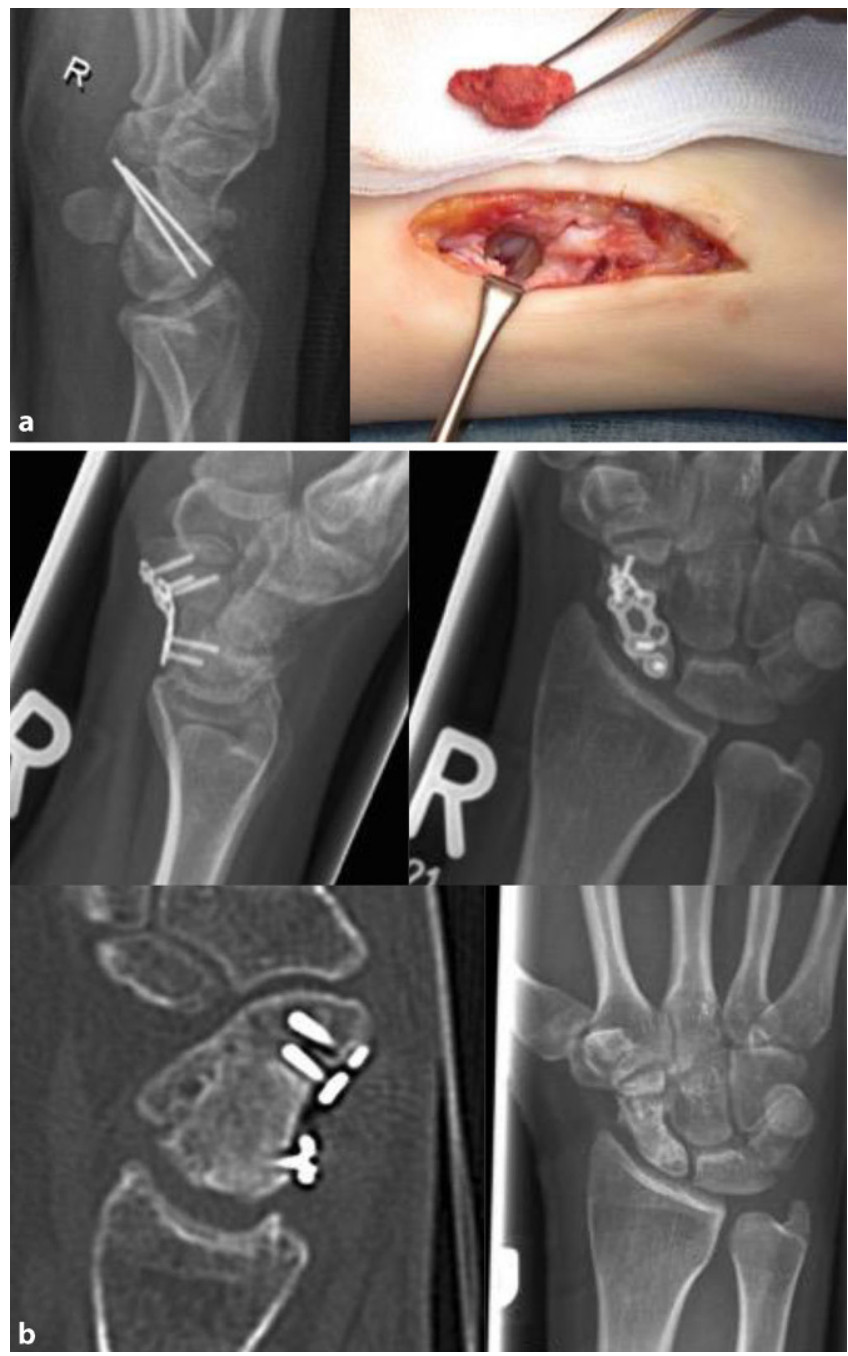

Abb. $2 \triangleleft$ Fallbeispiel 2. a Pseudarthrose nach fehlgeschlagener, auswärtiger Osteosynthese: operative Revision, Entfernung der Kirschner-Drähte und Ausräumung der Pseudarthrose sowie Rekonstruktion mittels Beckenkammspan. b Die Osteosynthese erfolgt hier mittels winkelstabiler Skaphoidplatte (Medartis AG, Basel, Schweiz). Die Materialentfernung erfolgte nach knöcherner Heilung
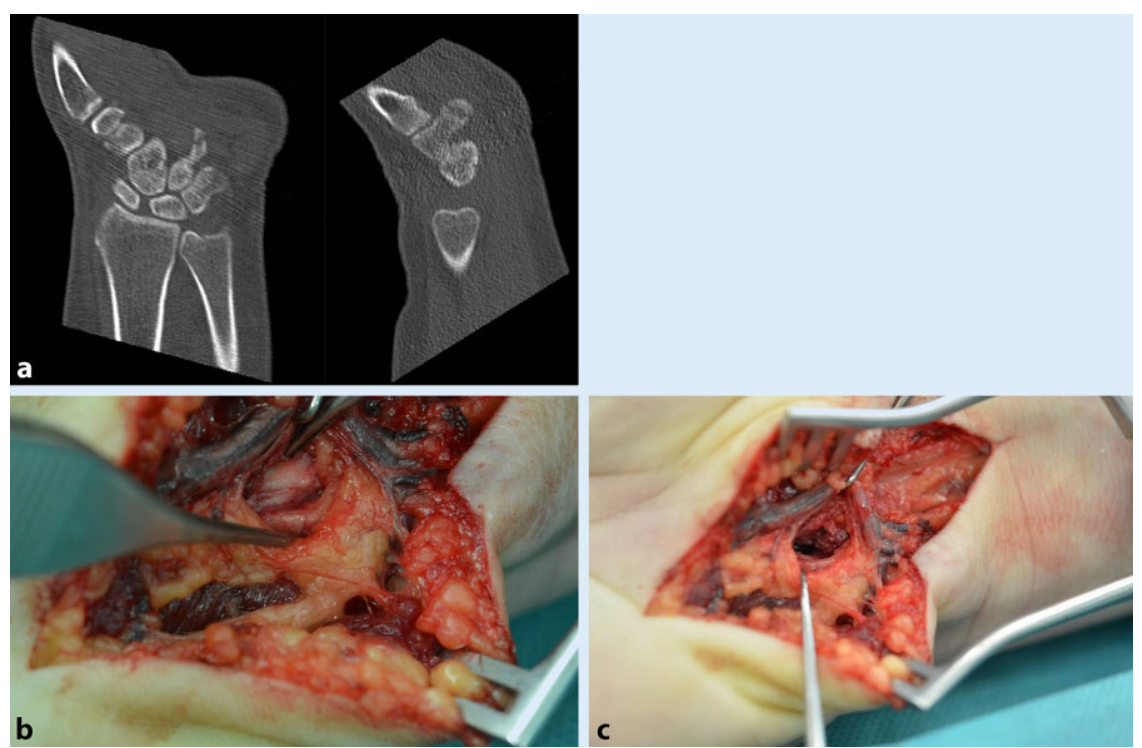

Abb. 3 A Fallbeispiel 3.a 46-jährige Patientin mit Pseudarthrose des Hamulus ossis hamati nach Sturz auf den rechten Arm vor 3 Monaten. b Intraoperative Aufnahme mit Spaltung der Loge de Guyon, Neurolyse des N. ulnaris und Darstellung des Hamulus ossis hamati. c Intraoperative Aufnahme nach Resektion des Hamulus ossis hamati
Ausräumung der Pseudarthrose, Auffüllung des knöchernen Defektes mittels Spongiosaplastik und Osteosynthese mittels stabiler Osteosynthese (meist winkelstabile Plattenosteosynthese). Bei der atrophen Pseudarthrose besteht ein Substanzdefekt. Auch hier ist eine operative Intervention notwendig. Nach der Pseudarthroseausräumung ist je nach Defektgröße eine Spongiosaanlagerung oder ein kortikospongiöser Beckenkammspan notwendig (Fallbeispiel 4, - Abb. 4a-c). Bei größeren Defekten ist ggf. auch ein freier vaskularisierter Knochentransfer indiziert. Daran schließt sich ebenfalls eine stabile Osteosynthese an.

Zusätzliche Maßnahmen sowohl bei karpalen als auch bei metakarpalen Pseudarthrosen sind nach erfolgter operativer Intervention die niedrigenergetische Ultraschalltherapie sowie die Möglichkeit der extrakorporalen Stoßwellentherapie, die die knöcherne Heilung unterstützen sollen $[15,18,19]$.

\section{Fazit für die Praxis}

- Bei den karpalen und metakarpalen Pseudarthrosen handelt es sich um eine multifaktorielle Erkrankung.

- Häufig entstehen die Pseudarthrosen aufgrund übersehener oder nicht adäquat behandelter karpaler oder metakarpaler Frakturen.

- Die Skaphoidpseudarthrose ist die häufigste Pseudarthroseform am Karpus.

- Neben der klinischen Symptomatik ist eine suffiziente bildgebende Diagnostik zwingend notwendig.

- Zur besseren Beurteilung ist eine CTUntersuchung zu fordern, ggf. auch eine MRT-Untersuchung.

- Die Therapie besteht in der operativen Ausräumung der Pseudarthrose, der knöchernen Auffüllung als formwiederherstellendes Verfahren mit Erreichen einer suffizienten Stabilität.

- Revaskularisierende Verfahren sind bei Minderperfusion oder nach fehlgeschlagenen Voroperationen, aber auch bei bestehenden größeren Defekten notwendig. 

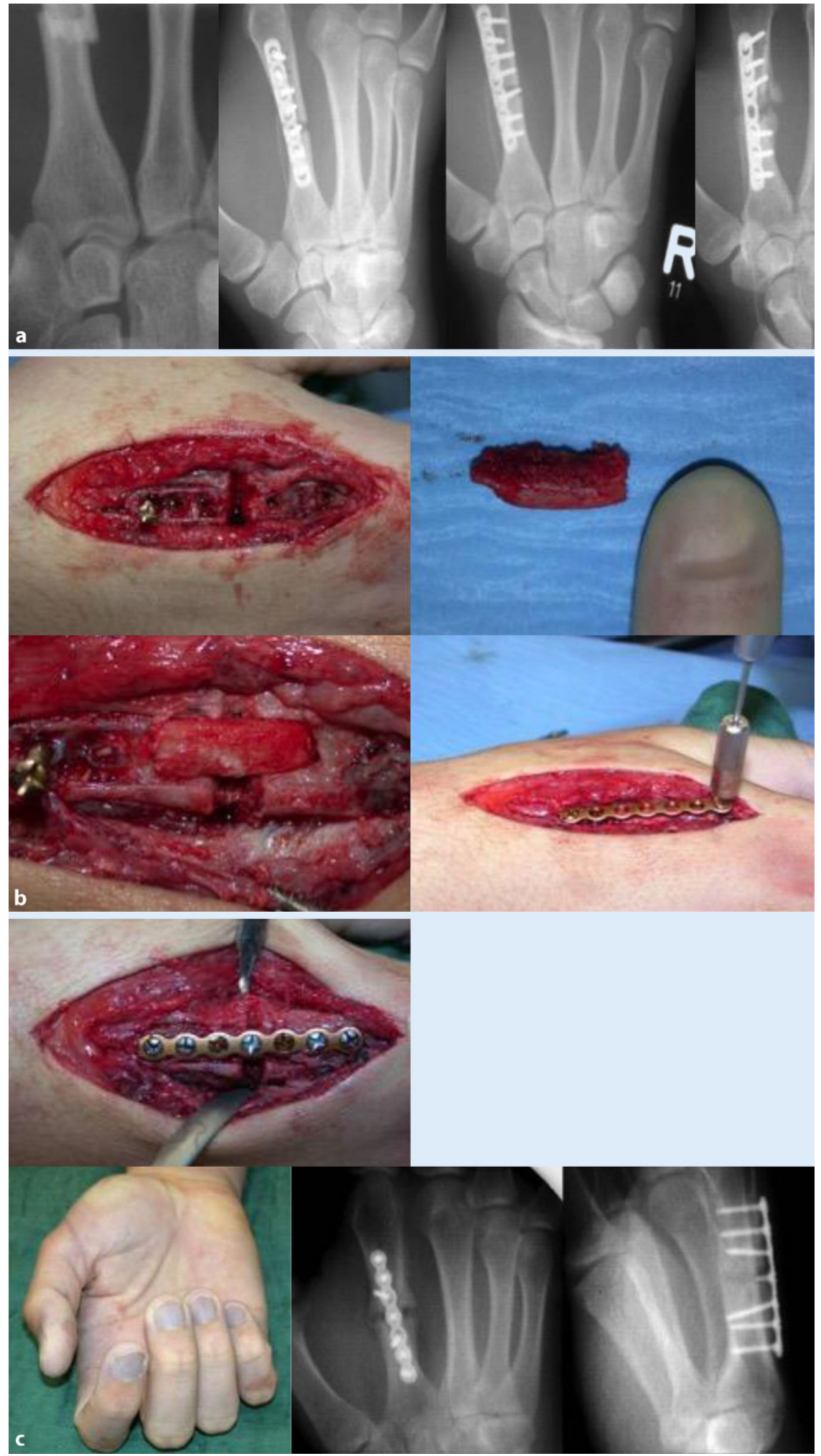

Abb. 4 ム Fallbeispiel 4.Pseudarthrose 2. Mittelhandknochen. a Auswärtige Erstversorgung einer Mittelhandknochen-II-Fraktur mit Fragmentstellung auf Distanz und Schraube im Frakturspalt; auswärtige Reosteosynthese auf Distanz ohne Spongiosaplastik. Klinisch zeigten sich ein Rotationsfehler sowie radiologisch eine Pseudarthrose. $\mathbf{b}$ Erneute Reosteosynthese mit kortikospongiösem Beckenkammspan - intraoperative Bilder. c Intraoperatives Bild sowie radiologische postoperative Kontrolle: Span mit monokortikaler Schraube gefasst. Das klinische Foto zeigt die korrekt eingestellte Rotation

\section{Korrespondenzadresse}

\section{Dr. A. Arsalan-Werner}

Abteilung für Plastische, Hand- und Rekonstruktive Chirurgie, Berufsgenossenschaftliche Unfallklinik Frankfurt am Main

Friedberger Landstr. 430, 60389 Frankfurt am Main, Deutschland pc-hc@bgu-frankfurt.de

\section{Einhaltung ethischer Richtlinien}

Interessenkonflikt. M. Sauerbier hat einen Beratervertrag mit der Medartis AG, Basel, Schweiz. I.M. Mehling und A. Arsalan-Werner geben an, dass kein Interessenkonflikt besteht.

Dieser Beitrag beinhaltet keine von den Autoren durchgeführten Studien an Menschen oder Tieren.

The supplement containing this article is not sponsored by industry.

\section{Literatur}

1. Baumeister HH, Greinemann H (1989) Zur konservativen Behandlung des Kahnbeinbruches der Handwurzel. Unfallchirurg 92:175-179

2. Bickert B, Kremer T, Kneser U (2014) Operative Therapie der Skaphoidpseudarthrose. Obere Extremität 9:271-275

3. Brauer RB, Dierking M, Werber KD (1997) Herbert-Schraube mit der Freehand-Methode zur Osteosynthese der frischen Skaphoidfraktur. Unfallchirurg 100:776-781

4. Choudry UH, Bakri K, Moran SL et al (2008) The vascularized medial femoral condyle periosteal bone flap for the treatment of recalcitrant bony nonunions. Ann Plast Surg 60:174-180

5. Dias JJ, Taylor M, Thompson J et al (1988) Radiographic signs of union of scaphoid fractures. An analysis of inter-observer agreement and reproducibility. JBone Joint Surg Br 70:299-301

6. Gabl M (2015) Karpale Pseudarthrosen. In: Sauerbier Metal (Hrsg) Die Handchirurgie. Elsevier Urban \& Fischer, München, S 252-263

7. Harhaus L, Bickert B, Kneser U (2016) Pseudarthrosen und komplikationsbehaftete Verläufe nach Mittelhandfrakturen. Trauma Berufskrankh 18(Suppl4):372-375

8. Herbert TJ (1990) The fractured scaphoid. Quality Medical Publishing, St. Louis

9. Herbert TJ, Filan SL (1999) Osteosynthese von proximal gelegenen Kahnbeinpseudarthrosen. Handchir Mikrochir Plast Chir 31(3):169-173

10. Hertel R, Masquelet AC (1989) The reverse flow medial knee osteoperiosteal flap for skeletal reconstruction of the leg. Description and anatomical basis. Surg Radiol Anat 11:257-262

11. Jones DB Jr., Burger H, Bishop AT et al (2008) Treatment of scaphoid waist nonunions with an avascular proximal pole and carpal collapse. A comparison of two vascularized bone grafts. JBone Joint Surg Am 90:2616-2625

12. Jones DB Jr., Burger H, Bishop AT et al (2009) Treatment of scaphoid waist nonunions with an avascular proximal pole and carpal collapse. 
Surgical technique. J Bone Joint Surg Am 91 (Suppl 2):169-183

13. Jones DB Jr., Moran SL, Bishop AT et al (2010) Freevascularized medial femoral condyle bone transfer in the treatment of scaphoid nonunions. Plast Reconstr Surg 125:1176-1184

14. Just-Kovac L, Innocenti M, Sauerbier M (2011) Möglichkeiten und Ergebnisse der (freien) vaskularisierten Knochentransplantation im Handbereich. Obere Extremität 6:189-198

15. Malizos KN, Hantes ME, Protopappas V et al (2006) Low-intensity pulsed ultrasound for bone healing: an overview. Injury 37(Suppl 1):S56-S62

16. Mehling IM, Sauerbier M (2015) Der posttraumatische karpale Kollaps: SLAC- und SNAC-Wrist. Handchir Scan 04(02):137-152

17. Preisser $P$ (2003) Operative Behandlung von Pseudarthrosen an Mittelhand und Phalangen. Trauma Berufskrankh 5:92-97

18. RubinC,BolanderM, RyabyJPetal (2001)Theuse of low-intensity ultrasound to accelerate the healing of fractures. J Bone Joint Surg Am 83:259-270

19. Watanabe Y, Matsushita T, Bhandari M et al (2010) Ultrasound for fracture healing: current evidence. J Orthop Trauma 24(Suppl 1):S56-S61

20. Yamamoto H, Jones DB Jr., Moran SL et al (2010) The arterial anatomy of the medial femoral condyle and its clinical implications. J Hand Surg Eur Vol 35:569-574

21. Zaidemberg C, Siebert JW, Angrigiani C (1991) A new vascularized bone graft for scaphoid nonunion. J Hand Surg Am 16:474-478 